\title{
Quantification of the minimum amount of lemon juice and apple cider vinegar required for the growth inhibition of dandruff causing fungi Malassezia furfur
}

\author{
PV Parvati Sai Arun ${ }^{1 *}$, Yaralagadda Vineetha ${ }^{2}$, Maseera Waheed ${ }^{3}$, Koka Ravikanth ${ }^{4}$ \\ 1,2,3,4 Department of Biotechnology, Chaitanya Bharathi Institute of Technology, Gandipet, Hyderabad, India \\ *Corresponding Author: arun.uoh@gmail.com, Tel.: +91-70751-11851
}

Available online at: www.isroset.org

Received: 02/Apr/2019, Accepted: 13/Apr/2019, Online: 30/Apr/2019

\begin{abstract}
The main focus of this study is to quantify the amount of lemon juice and apple cider vinegar required to inhibit the growth of lipophilic fungus, Malassezia furfur. Malassezia furfur infects the human scalp, affects hair and causes the dandruff. In this work, the antifungal effect of lemon and apple cider vinegar was investigated along with commercially available antifungal drug fluconazole. Agar diffusion method was used and the zone of inhibition was measured to quantify the amount required and also to estimate the antifungal efficacy of lemon juice and apple cider vinegar. We have used different concentration of lemon juice and apple cider vinegar such as 10\%, 20\%, 30\%, 40\%, and 50\% concentrations. From our results, by observing the zones of inhibitions on the petridishes, we confirm that lemon juice and apple cider vinegar has good antifungal effect on Malassezia furfur and around $3 \mathrm{ml}$ to $4 \mathrm{ml}$ of lemon juice or apple cider vinegar diluted and made to $10 \mathrm{ml}$ of the solution with water would be sufficient enough to inhibit the growth of Malassezia furfur. This study justifies the use of either lemon juice or apple cider vinegar as a remedy for Malassezia furfur infected scalp and hair.
\end{abstract}

Keywords-Malasezzia furfur, lemon juice, apple cider vinegar, antifungal activity

\section{INTRODUCTION}

Dandruff is a condition of the skin on head, the scalp, where its appearance is restricted to scalp and is characterized by itching and excessive flaking of the skin on the scalp[1]. It is easy to detect dandruff as it appears as small, round, whiteto-gray patches on the scalp. Dandruff is also considered as the most commercially important and exploited skin disease [2]. It has been reported that human being definitely suffer with dandruff problem at some point of their life time [3]. Of many causes that lead to the condition of dandruff, one of the key causative factors that cause dandruff is fungal infection. The fungus, Malassezia furfur, a lipophilic fungus infects the healthy scalp leads to the dandruff condition [4]. Malassezia furfur is a kind of yeast which was initially named as Pityrosporum ovale[6], which upon infection on the scalp, it feed on the scalp lipids and proteins and finally leads to the increase of lipase activity. The increased lipase activity leads to hypersensitive reactions due to the release of free fatty acids [5]. There are many chemical derivatives used for the purpose of management of dandruff such as glycolic acids, salicylic acid, zinc pyrithione, sulphur and also tar derivative [4]. There are also some naturally derived compounds from plants which are used for the management of the dandruff, such as sheath and gel of Aloe babadensis, leaves of Hibiscus rosasinensis, leaves of Lawsonia inermis and many other plant and their body parts[5]. Previously, the growth inhibitory effect of lemon and other plant extracts was demonstrated elsewhere[7], but to the best of our knowledge, quantification of minimum amount of lemon juice or apple cider vinegar required for growth inhibition of Malassezia furfur was not reported. Thus, in this report we aim to quantify the amount of lemon juice or apple cider vinegar required for growth inhibition of Malassezia furfur using standard methods.

\section{METHODOLOGY}

\section{Revival of the strain and maintaining the culture}

We obtained the pure culture of Malassezia furfur MTCC 1374 from Institute of Microbial Technology (IMTECH) Chandigarh, India. The obtained culture was revived in the following manner. Initially, we autoclaved about 30 petridishes necessary for our experiment. Later, we prepared Emmon's modified Sabouraud's dextrose media for the revival of the fungal strain in two sets. In the first set, we used the following ingredients such as dextrose 40g/l, peptone $10 \mathrm{~g} / \mathrm{l}$, agar $17 \mathrm{~g} / \mathrm{l}$, and we added distilled water to make up the solution to one liter. The second set was similar to the first set but it does not have agar as its ingredient 
(liquid broth). The $\mathrm{pH}$ of the both media were adjusted to 6 using HCL at $25^{\circ} \mathrm{c}$ degrees and then set for sterilization using autoclave. After sterilization, the first set of media was poured in to petridishes and allowed for solidification. From the second set of media i.e the liquid broth, we took about $20 \mu \mathrm{l}$ of the liquid broth and added to the lyophilized culture which was obtained from IMTECH and mixed gently by shaking (hereafter this mixture is referred as the sample). After 20 minutes, we have taken a sterile inoculation loop and dipped in the sample and streaked in one of the solidified petridish and labeled. The said petridish was kept in incubation chamber at room temperature and incubated for 3 days. After 3 days of incubation, we have transferred a loop full of fugal culture from the petridish to liquid broth and set for incubation as described earlier.

\section{Estimation of minimum amount of lemon juice and apple cider vinegar required for growth inhibition of Malassezia furfur}

Emmon's modified Sabouraud's dextrose media was prepared a fresh as described earlier and set for sterilization using autoclave. Fresh and sterile petridishes were taken and the sterilized media was added to them and allowed for solidification. Once the media in the petridishes gets solidified, about $400 \mathrm{ml}$ of culture was spreaded on the petridishes and allowed it to dry for 15 minutes. After 15 minutes, two deep wells were made on the solidified media separated apart at the corners of the petridish, which can accommodate about $100 \mu \mathrm{l}$ of lemon juice and apple cider vinegar. We also made a paper disc of $5 \mathrm{~mm}$ diameter and place on the third corner of the petridish and added about $10 \mathrm{mcg}$ of fluconazole anti-fungal drug which was obtained commercially. In this way a total of 5 petridishes, which vary in the concentrations of the lemon juice and apple cider vinegar were were taken.(lemon juice and apple cider vinegar are hereafter referred as substrates). We have taken substrates concentrations as $1 \mathrm{ml}$ substrate in $9 \mathrm{ml}$ water, followed by $2 \mathrm{ml}$ in $8 \mathrm{ml}$ water, $3 \mathrm{ml}$ in $7 \mathrm{ml}$ water, $4 \mathrm{ml}$ in $6 \mathrm{ml}$ of water, $5 \mathrm{ml}$ in $5 \mathrm{ml}$ of water respectively for both lemon juice and apple cider vinegar. Fluconazole concentration was kept constant in all the 5 petridishes. Later, the five petridishes which contain the lemon juice, apple cider vinegar and fluconazole disc along with the Malassezia furfur culture were kept under incubation to allow the fungi to grow at room temperature for week days.

\section{RESULTS AND DISCUSSION}

Revival of Malassezia furfur estimation of minimum amount of substrates required for growth inhibition

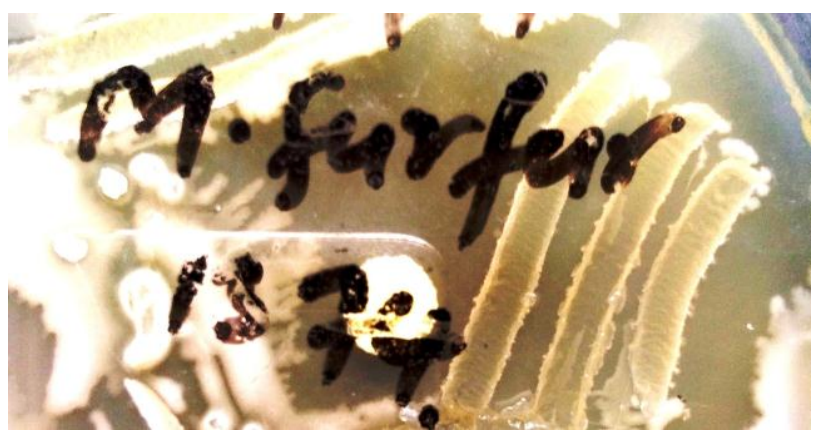

Figure 1: Revival of Malassezia furfur on Emmon's modified Sabouraud's dextrose media after three days of incubation.

After three days of incubation, we found a noticeable growth of Malassezia furfur on the streaked petridish (Figure 1). As described in materials and methods, we later transferred this culture in liquid broth of Emmon's modified Sabouraud's dextrose medium for further analysis. The petridishes with the fungal culture, along with different concentrations of substrates were examined for the zone of inhibition (Figure 2). The zone of inhibition values for each petridish was as follows. Table 1 shows the zone of inhibition values of

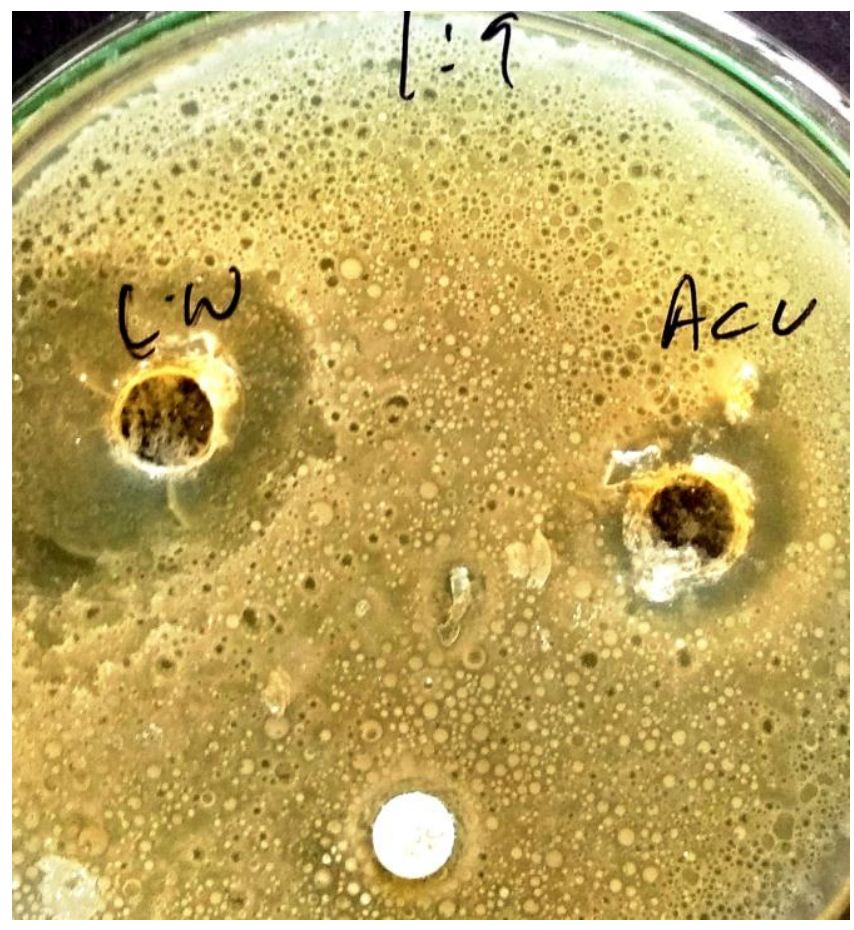

Figure 2: Zone of inhibition of the substrates with concentration of $1 \mathrm{ml}$ substrate in $9 \mathrm{ml}$ of distilled water (1:9). In this snapshot, L.W represents the well on the gel with lemon juice and on the right hand side ACV represents the well with apple cider vinegar. The white colour disc represents the disc with $10 \mathrm{mcg}$ fluconazole. We can clearly the efficacy of each substrate used in the study. Lemon juice has more zone in this sample than that of the other two substrates.

each substrate with different concentration. We have ignored the zone of inhibition of fluconazole, since it showed very 
minor zone of inhibition in all the samples compared as shown in Figure 2. We have observed the zone of inhibition to be increasing in the diameter as the concentration of the substrates increases. We have found the maximum zone of inhibition of Malassezia furfur in the petridish which has substrate and water in 1:1 ration $(5 \mathrm{ml}$ of substrate in $5 \mathrm{ml}$ of water) (Figure 3).

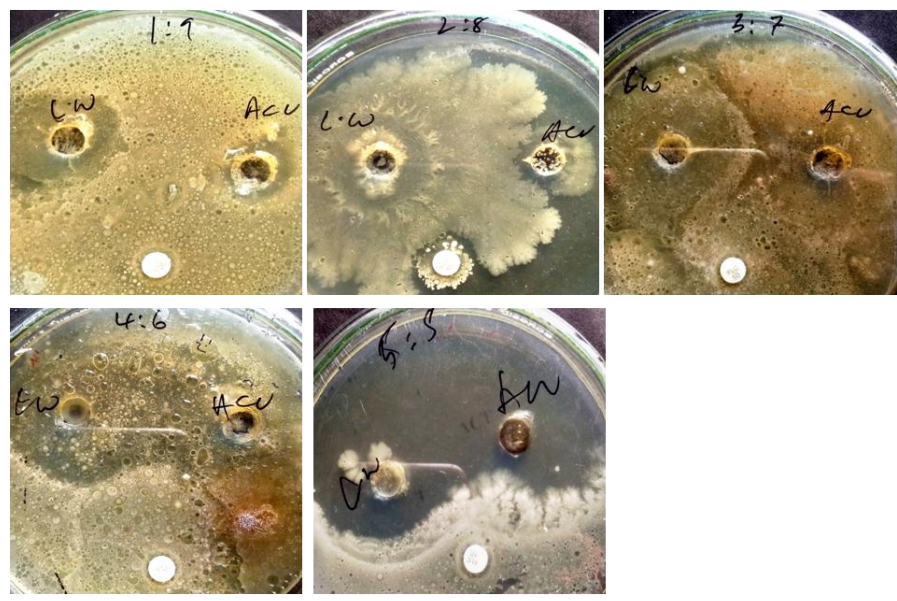

Figure 3: Zone of inhibition of the substrates with different concentration. In this snapshot, L.W represents the well on the gel with lemon juice and on the right hand side ACV represents the well with apple cider vinegar. The white colour disc represents the disc with $10 \mathrm{mcg}$ fluconazole. We can clearly the efficacy of each substrate used in the study. Lemon juice has more zone of inhibition than that of apple cider vinegar in the 2:8 dilution. But in later concentrations, it is found to be normal.

Table 1: Table showing the information of zone of inhibitions of each substrate on Malassezia furfur

\begin{tabular}{|c|c|c|}
\hline $\begin{array}{c}\text { Concentration } \\
\text { Substrate : Water }\end{array}$ & $\begin{array}{c}\text { Zone of } \\
\text { inhibition of } \\
\text { Lemon } \\
\text { Juice in } \mathbf{~ m m}\end{array}$ & $\begin{array}{c}\text { Zone of } \\
\text { inhibition of } \\
\text { Apple cider } \\
\text { vinegar in } \mathbf{~ m m}\end{array}$ \\
\hline $1: 9$ & 12 & 8 \\
\hline $2: 8$ & 12 & 9 \\
\hline $3: 7$ & 16 & 14 \\
\hline $4: 6$ & 17 & 15 \\
\hline $5: 5$ & 25 & 23 \\
\hline
\end{tabular}

From the above zone of inhibition values we have calculated the minimum amount of lemon juice or apple cider vinegar required for inhibition of Malassezia furfur with the following equation

$$
A=\frac{1}{\mathrm{n}} * \sum_{i=1}^{n}\left(x_{i}\right)
$$

Where $\mathrm{A}=$ Average.

$\mathrm{n}=$ Total number of observations.

$x_{i}=$ Value of individual zone of inhibition.
From this equation, we found that the minimum amount of lemon juice and apple cider vinegar required for the inhibition of Malassezia furfur and dandruff was found to be $3-4 \mathrm{ml}$ made up to $10 \mathrm{ml}$ water.

\section{CONCLUSION AND FUTURE SCOPE}

This preliminary report is first of its kind to quantify the amount of lemon juice and apple cider vinegar required for growth inhibition in dandruff causing fungi Malassezia furfur. This work is preliminary in nature and performed to understand the inhibitory effect of lemon and apple cider vinegar M.furfur and to quantify them. Moreover from our findings, we also conclude that the use of Fluconazole which is commercially abavilable in the marked in $10 \mathrm{mcg}$ concentration is less effective than that of plant derived products, such as lemon juice and apple cider vinegar on M.furfur. This work also has future scope in such a way that to characterise the medicinal factors or the constituents of lemon juice and apple cider vinegar, which are the causative factors for the inhibition of the fungi using different chromatography techniques.

\section{ACKNOWLEDGMENT}

We thank Deparment of Biotechnology, Chaitanya Bharathi Institute of Technology, for providing the laboratory facilities to perform this project.

\section{Funding Information}

None

\section{Author Contributions}

PVPSA conceived the experiments, analyzed and wrote the paper, COB analyzed the paper and participated actively in discussions, $\mathrm{MW}$ and $\mathrm{KR}$ involved in conducting the experiments, $\mathrm{YV}$ involved involved experiments and data generation.

\section{REFERENCES}

\section{Referencese}

[1]. J.B Luis J, C. T.C Wikramanayake, "Seborrheic dermatitis and dandruff: a comprehensive review." Journal of clinical and investigative Dermatology, 3(2), 2015.

[2]. S.Ranganathan, T. Mukhopadhyay, "Dandruff: the most commercially exploited skin disease." Indian journal of Dermatology, 55(2),130, 2010.

[3]. A.K. Gupta, R. Batra, R.Bluhm, T. Boekhout, T.L. Dawson Jr, "Skin diseases associated with Malassezia species." Journal of the American Academy of Dermatology, 51(5), 785-798, 2004.

[4]. R. Vijayakumar, C. Muthkumar, T. Kumar, R. Sarvanamuthu, "Characterization of Malassezia furfur and its controlby using plant extracts", Indian journal of Dermatology, 51(2), 145-148, 2006. 
[5]. S. Shuster, "The aetiology of dandruff and the mode of action of therapeutic agents". British journal of Dermatology, 111, 235-242, 1984.

[6]. J.. Faergemann, J.C. Jones, O. Hettler, Y. Loria, "Pityrosporum ovale (Malassezia furfur) as the causative agent of seborrhoeic dermatitis: New treatment options." British Journal of Dermatology, 134 :Suppl. 46, 12-15, 1996

[7]. M. Pingili, S. Vanga, R.K. Raparla, "Antifungal activity of plant extracts against dandruff causing organism Malassezia furfur." International Journal of Bioassays, 5(11), 5047-9, 2016.

\section{AUTHORS PROFILE}

Dr. PV Parvati Sai Arun has pursed B. Tech in Biotechnology from JNTU, M.Tech in Bioinformatics, and Ph.D in Functional Genomics from University of Hyderabad in 2007,2009 and 2015. $\mathrm{He}$ is currently working as Assistant Professor in Department of Biotechnology from Chaitanya Bharathi Institute of Technology (CBIT), Hyderabad since 2018. He has published more than 12 research papers in reputed international journals including Thomson Reuters (SCI, Scopus \& Web of Science) which are available online. His main research work focuses on Computational biology, Statistical Genomics and NGS. 